\title{
FUNCTIONAL ASSESSMENT USING CONSTANT'S SHOULDER SCALE AFTER MODIFIED RADICAL AND SELECTIVE NECK DISSECTION
}

\author{
Douglas B. Chepeha, MD, ${ }^{1}$ Rodney J. Taylor, MD, ${ }^{1}$ Judith C. Chepeha, MScPT, ${ }^{2}$ \\ Theodoros N. Teknos, MD, ${ }^{1}$ Carol R. Bradford, MD, ${ }^{1}$ Pramod K. Sharma, MD, ${ }^{1}$ \\ Jeffrey E. Terrell, MD, ${ }^{1}$ Gregory T. Wolf, MD ${ }^{1}$ \\ ${ }^{1}$ Department of Otolaryngology-Head \& Neck Surgery, University of Michigan, 1500 E. Medical Center Drive, \\ Taubman Center 1904, Ann Arbor, Michigan 48109-0312. E-mail: dchepeha@umich.edu \\ ${ }^{2}$ Department of Physical Therapy, Glen Sather Sports Medicine Clinic, University of Alberta, Edmonton, \\ Alberta, Canada
}

Accepted 2 November 2001

Published online 11 February 2002 in Wiley InterScience (www.interscience.wiley.com).

DOI: $10.1002 /$ hed.10067

\begin{abstract}
Background. Constant's Shoulder Scale is a validated and widely applied instrument for assessment of shoulder function. We used this instrument to assess which treatment and demographic variables contribute to shoulder dysfunction after neck dissection in head and neck cancer patients.

Methods. A convenience sample of 54 patients with 64 neck dissections and minimum follow-up of 11 months were evaluated. Thirty-two accessory nerve-sparing modified radical (MRND) and 32 selective neck (SND) dissections were performed. Multivariable regression analysis was used to determine the variables that were predictive for shoulder dysfunction. Clinical variables included age, time from surgery, handedness, weight, radiation therapy, neck dissection type, tumor stage, and site.

Results. Patients receiving MRND had significantly worse shoulder function than patients with SND $(p=.0007)$. Radiation therapy contributed negatively, whereas weight contributed positively $(p=.0001)$.

Conclusions. The critical factors contributing to shoulder dysfunction after neck dissection were weight, radiation therapy, and neck dissection type. (c) 2002 Wiley Periodicals, Inc. Head Neck 24: 432-436, 2002
\end{abstract}

Correspondence to: D. B. Chepeha

(๑) 2002 Wiley Periodicals, Inc.
Keywords: quality of life; radical neck dissection; selective neck dissection; shoulder; neck

To determine the factors that are predictive of long-term shoulder dysfunction after neck dissection, we conducted a cross-sectional study of postoperative patients who previously received a neck dissection as part of the management of their head and neck cancer. Empirically, many patients often recover shoulder function after a neck dissection with little or no consequence, whereas others have protracted shoulder disability. We endeavored to study the factors that most contribute to long-term shoulder dysfunction for patients having received selective neck dissections (SND) and modified radical neck dissections (MRND) sparing the accessory nerve.

It is well established that neck dissection procedures are associated with shoulder dysfunction. Nahum and Marmor. ${ }^{1}$ First described the "shoulder syndrome" resulting from radical 
neck dissection, which is characterized by shoulder pain, limitations of abduction, and scapular winging. ${ }^{1}$ Modifications of the radical neck dissection (RND) were fashioned to limit the extent and frequency of shoulder dysfunction that resulted from the RND. ${ }^{2,3}$ Nevertheless, significant shoulder dysfunction continues to arise even with accessory nerve-sparing neck dissection procedures. ${ }^{4,5}$

Because of its established validity and reliability, the Constant's Shoulder Test was used to provide a clinical assessment of patient's shoulder function. ${ }^{6}$ It was our goal to determine which clinical and demographic determinants were important to long-term shoulder impairment. Specifically, we were interested to ascertain the contribution to shoulder impairment of factors such as type of neck dissection, radiation therapy, disease extent and site, and time from surgery. We hypothesized that patients having received SNDs would demonstrate significantly less shoulder impairment than those having had modified radical neck dissections sparing the accessory nerve.

\section{MATERIALS AND METHODS}

Study Design. We conducted a cross-sectional study to evaluate the factors that have an impact on shoulder function after neck dissection procedures. Our intention was to assess long-term shoulder function after neck dissection procedures, where the accessory nerve was preserved (SND and MRND).

The population was derived from consecutive head and neck cancer patients who were seen at the University of Michigan Department of Otolaryngology outpatient clinic. Patients were accrued until an approximately equal number of patients with SND and MRND were obtained. Sample size calculations were based on the mean difference in scores on the pain domain of the University of Michigan Head and Neck QOL questionnaire for SND and MRND. ${ }^{7}$ The data verified that approximately 32 neck dissection procedures in each group would be adequate to show a statistical difference between the two neck dissection groups.

On study entry, informed consent was obtained, and Constant's Shoulder Functional Test was carried out on each patient. For patients with bilateral neck dissections, each side was evaluated and scored separately with Constant's Shoulder Test.
Patients. There were 54 patients in the study group, accounting for a total of 64 neck dissection procedures (10 patients received bilateral procedures). A total of 32 accessory nerve-sparing modified radical neck dissections and 32 selective neck dissection procedures were performed in this group. The average age of the study population was $56.9 \pm 11.8$ years. Seventy-four percent of the group were men, and the mean time elapsed from surgery was 33.7 months (range, 11-120 months), 22.4 months, and 42.9 months for SND and MRND, respectively. The difference in time interval between SND and MRND is that, until recent years, MRND was the standard neck dissection procedure performed in our patients for clinically negative or limited neck disease. Now, at our institution, SND is replacing MRND for some indications. Therefore, most of the patients with MRND have a longer duration of follow-up. Most patients received both surgery and radiation therapy (87\%), and squamous cell carcinoma was the most common tumor histologic finding (92.5\%) (Table 1).

Patient inclusion criteria were those who were previously untreated with a diagnosis of head and neck cancer and concurrently required a SND or MRND. All SND procedures were performed as described by Medina, ${ }^{3}$ and none included dissection of level $\mathrm{V}$ or excluded dissection of levels II and III. At our institution, each pa-

\begin{tabular}{lcc}
\hline \multicolumn{2}{l}{ Table 1. Clinical demographic data by neck dissection type. } \\
\hline Clinical/demographics & SND & MRND \\
\hline Age (y) & 56.0 & 57.6 \\
Weight (kg) & 75.3 & 85.6 \\
Time from surgery, (m) & 22.4 & 42.9 \\
Constant's score & 80.1 & 62.8 \\
Number of neck dissections & 32 & 32 \\
Tumor site & & \\
Oral cavity & 6 & 4 \\
Oropharynx & 10 & 13 \\
Larynx/hypopharynx & 4 & 6 \\
Unknown primary & 2 & 1 \\
Other & 3 & 5 \\
T stage & & \\
Tx & 2 & 2 \\
T1,T2 & 9 & 6 \\
T3,T14 & 4 & 21 \\
N stage & & \\
N0 & 20 & 10 \\
N1 & 5 & 5 \\
N2 & 7 & 16 \\
N3 & 0 & 1 \\
\hline
\end{tabular}

Abbreviations: MRND, modified radical neck dissection; SND, selective neck dissection. 
tient in this study with a selective neck dissection received an identical accessory nerve dissection. The investing fascia is elevated off the medial aspect of the SCM until the accessory nerve is identified. Then the cervical rootlets are identified more posteriorly. The accessory nerve is dissected from all surrounding tissue from its entry into the sternocleidomastoid (SCM) to the skull base. The entire submuscular triangle and contents of level II are resected with the neck dissection specimen. The cervical rootlets serve as the posterior margin of our SND. The dissection is brought forward along the rootlets, separating both levels III and IV from level V. This approach in this region results in a very thorough posterior jugular dissection. Regardless of the patient's weight, the technical approach to each neck dissection was identical. In our population, we did not compromise the identification or dissection of the accessory nerve or the cervical rootlets.

Patients were excluded if they were not at least 11 months from surgery or reported any history of unrelated neck or shoulder disease. Patients who enrolled received their head and neck cancer treatment at the University of Michigan in the Department of Otolaryngology and were encountered on an outpatient basis during their routine follow-up care. The University of Michigan's Institutional Review Board approved study materials and methods.

Constant's Shoulder Assessment. Constant's Shoulder Test is a validated clinical assessment of shoulder function that has established usefulness and accuracy across many diseases affecting the shoulder. This validated, widely used clinical test has been shown to be an accurate and sensitive measure of shoulder function, detecting subtle changes in shoulder function. ${ }^{6,8}$ The assessment takes approximately 10 minutes to perform. It is a weighted test that combines patient symptom scores (35\%) and objective measures of active shoulder function (65\%). The symptoms score assesses pain, sleep, recreation, and vocational activities. The objective score measures active shoulder range of motion, combined internal rotation, combined external rotation, and shoulder strength in the plane of the scapula. Scores range from 0 to 100, with higher scores indicating better shoulder function. A single individual (RJT) performed each assessment.
Statistical Methods. All patient data, including demographic information, survey data, and disease information, were stored in a relational database on a Macintosh G3 personal computer. Data entries were checked and verified against the primary data. All statistical analyses were done using SAS Institute Software, version 6.12 (Carey, NC).

Multivariable regression analysis was used to model the dependent variables. Univariate and stepwise regression analyses were used to facilitate selection of variables. The variables also were evaluated for the presence of multicollinearity, such that candidate models contained no violations of multicollinearity. Independent variables assessed included patient age, gender, time elapsed from surgery, tumor stage (T1-2 or T3-4), tumor site (oral cavity, pharynx and hypopharynx, and larynx), patient weight, radiation therapy, handedness, and neck dissection type (SND or MRND).

Certainly, we considered that the difference in time interval might have an impact. It was our concern that those patients further from surgery might have improved rather than reduced shoulder function compared with those who had surgery more recently. [All patients were followed a minimum of 11 months.] We performed a multiple variable regression analysis to assess this possibility. The results of this analysis demonstrated that there was no statistical difference in shoulder function relative to the time from surgery.

\section{RESULTS}

For all patients, the mean score for Constant's Shoulder Test was $71.0 \pm 18.8$ (range, 22-100) with a standard deviation of 18.8. Raw subjective, objective, and total Constant's mean scores are categorized by both neck dissection type and radiation therapy are included in Tables 2 and 3 . We found that there was good correlation between Constant's subjective and objective measurements $(r=0.65, p<.0001)$.

We determined that when controlling for the clinical-demographic and treatment variables listed previously, patients with SND had statistically significantly better shoulder function than those having received MRND $(p=.0007)$. We also found that patient's weight was a significant factor as well $(p<.0001)$; more robust (ie, heavier) patients independently had better shoulder function than more diminutive patients after neck dissection procedures. Finally, the addition 
Table 2. Raw data constant's subjective and objective scores by neck

dissection type.

\begin{tabular}{lccc}
\hline Score & SND & MRND & $p$ value \\
\hline Constant's objective (0-65) & 50.8 & 40.8 & .0008 \\
Constant's subjective (0-35) & 29.1 & 22.0 & .0017 \\
Constant's total (0-100) & 79.9 & 62.8 & .0002 \\
\hline
\end{tabular}

Abbreviations: MRND, modified radical neck dissection; SND, selective neck dissection.

of radiation treatment was a critical factor in shoulder function after neck dissection. Although not statistically significant $(p=.1245)$ as an independent predictor, it was critical for the best model as determined by multiple variable linear regression.

The model equation that clearly emerged as the best predictor of shoulder function included the parameters: neck dissection type $(p=.0007)$, radiation therapy $(p=.1245)$, and patient's weight in kilograms $(p<.0001)$. The final model was Constant's score $=41.8-13.6^{*} N D-8.7 * X R T$ $+0.60 * \mathrm{~kg}$, $\left(\mathrm{R}^{2}=0.44\right)$. Several factors led to the selection of this model. The variables contained in this model were each found in the best one-, two-, and three-parameter models obtained with stepwise multivariable regression analysis. The addition of other clinical-demographic and treatment variables such as age, tumor site, time elapsed from surgery, handedness, and clinical stage did not significantly strengthen the regression model. Analysis for multicollinearity of the evaluated variables revealed no violations in multicollinearity.

For all patients, the mean score for Constant's Shoulder Test was 71.0 (range, 22-100), with a standard deviation of 18.8. The following is an example of how this model can be interpreted. For a $70-\mathrm{kg}$ patient, weight would contribute on average nearly 42 points $(0.60 * 70 \mathrm{~kg})$ to the overall score (maximum 100 points), although radiation therapy on average would decrease the overall score by 8.7 points, and $M R N D$ on average would decrease the score by 13.6 points. The intercept of constant, 41.8, would be added to obtain the score.

\section{DISCUSSION}

We used Constant's Shoulder Test is this study so that we could readily and reliably assess longterm shoulder impairment in an outpatient clinical setting. It was our goal to determine the factors that were most predictive of long-term shoulder impairment in this population. Among patients receiving neck dissections, those treated with SND had statistically better long-term shoulder function than those patients who received a MRND procedures did. This finding is consistent with Sobol's findings; he also found that patients receiving SND had objectively better physical measurements of shoulder function when assessed by range of motion, shoulder strength, and electromyographic measurements. ${ }^{4}$ However, there were limited long-term follow-up data available in their study, and their analysis did not control for potential confounding variables.

The critical difference between the SND and MRND is dissection in level V. Soo and Guiloff ${ }^{9}$ demonstrated that there is motor input from the cervical plexus to the trapezius through the accessory nerve. Even when these cervical contributions are preserved, there is usually some damage to the motor innervation of the muscles in the floor of the neck, which also may contribute to shoulder dysfunction. Still, if the deep neck fascia can be carefully preserved, the innervation of the trapezius is highly variable, and preservation of the accessory nerve and its related cervical nerve rootlets does not guarantee that all of the motor supply to the trapezius has been left intact. Even if the surgeon is technically superb during dissection in the posterior triangle, the unavoidable effect of devascularization and

Table 3. Raw data on constant's subjective and objective scores by radiation.

\begin{tabular}{lccc}
\hline Score & No radiation & Radiation & p value \\
\hline Constant's objective (0-65) & 51.4 & 44.5 & .12 \\
Constant's subjective (0-35) & 31.2 & 24.3 & .0046 \\
Constant's total (0-100) & 82.6 & 68.7 & .017 \\
\hline
\end{tabular}


scarring will likely lead to diminished shoulder function.

Our multiple variable regression analysis also demonstrated that weight was an important factor in long-term shoulder function. Heavier, more robust patients demonstrated significantly better shoulder function after neck dissection procedures, independent of other factors. Weight seemed to serve as a surrogate for overall physical well-being. In the model, weight was highly significant and accounted for a large magnitude of the overall equation for shoulder function. For a 70-kg patient, weight accounted for approximately $40 \%$ of the predicted calculation of shoulder function. It is possible that the more robust patients had better recuperative and compensatory powers, which translated into better preservation of shoulder function.

Finally, we found radiation therapy to be an important, but not statistically significant, negative contributor to long-term shoulder function after neck dissection. Schuller et $\mathrm{al}^{10}$ sought to evaluate the impact of both surgery and radiation on shoulder function. Their study found that the patients who received radiation in addition to surgery more frequently reported an increased reliance on others. Although an excellent study with important findings, the results needed to be confirmed with a validated questionnaire and an analysis that includes consideration of possible confounding clinical-demographic factors. We used Constant's Shoulder Test, a validated assessment of shoulder function, and radiation therapy emerged as an important, independent negative factor for long-term shoulder function. In a parallel study using a validated quality-oflife instrument, the Neck Dissection Impairment Index, we also found radiation to be an important, independent negative contributor to quality of life after neck dissection procedures. ${ }^{11}$

In this study, we determined the factors that most contributed to long-term shoulder function after neck dissection procedures. The type of neck dissection was found to be important; patients with SND had significantly better long-term function than patients with MRND. Also important was the protective effect of weight, which we believed was a surrogate for overall physical wellbeing and health status. Our results also suggest that the addition of radiation treatment is an important contributor to shoulder dysfunction. On the basis of the results of this study, MRND alone is more morbid than SND with postoperative radiation therapy. From a functional perspective, MRND should be considered only if the surgeon believes there is a clear oncologic advantage.

\section{REFERENCES}

1. Nahum AM, Marmor M. A syndrome resulting from radical neck dissection. Arch Otolaryngol 1961;74:82-86.

2. Boca E. Functional neck dissection: An evaluation and review of 843 cases. Laryngoscope 1984;94:942-945.

3. Medina J. Supraomohyoid neck dissection: Rational, indications, and surgical technique. Head Neck 1989;11: $111-122$.

4. Sobol S, Jensen C, Sawyer W. Objective comparison of physical dysfunction after neck dissection. Am J Surg 1985;150:503-509.

5. Leipzig B, Suen J, English J, Barnes J, Hooper M. Functional evaluation of the spinal accessory nerve after neck dissection. Am J Surg 1983;146:526-530.

6. Constant C, Murley G. A clinical method of functional assessment of the shoulder. Clin Orthop Rel Res 1987; 214:160-164.

7. Terrell J, Nanavati K. Head and neck cancer-specific quality of life. Arch Otolaryngol Head Neck Surg 1997; 123:1125-1132.

8. Van Den Ende C. Assessment of shoulder function in rheumatoid arthritis. The J Rheumatol 1996;23: 2043-2948.

9. Soo KC, Guiloff R. Innervation of the trapezius muscle: a study in patients undergoing neck dissections. Head Neck 1990;12:488-495.

10. Schuller D, Reiches N, Hamaker R, Lingeman R, Weisberger E, Conley J. Analysis of disability resulting from treatment including radical neck dissection or modified neck dissection. Head Neck Surg 1983;6:551-558.

11. Taylor R, Chepeha J, Teknos T, et al. Development and validation of the neck dissection impairment Index (NDII)-A quality of life measure. Presented at the American Academy of Otolaryngology - Head and Neck Surgery Foundation Annual Meeting. Washington, DC, September, 2000. 